\title{
Crystals from first principles
}

\section{A new calculation of the polymorphs of silica appears to have broken new ground in deriving crystal structure from chemical composition. But X-ray crystallographers need not worry - yet.}

ONE of the continuing scandals in the physical sciences is that it remains in general impossible to predict the structure of even the simplest crystalline solids from a knowledge of their chemical composition. Who, for example, would guess that graphite, not diamond, is the thermodynamically stable allotrope of carbon at ordinary temperature and pressure? Solids such as crystalline water (ice) are still thought to lie beyond mortals' ken.

Yet one would have thought that, by now, it should be possible to equip a sufficiently large computer with a sufficiently large program, type in the formula of the chemical and obtain, as output, the atomic coordinates of the atoms in a unit cell.

That time may not yet have arrived, but S. Tsuneyuki, M. Tsukada and H. Aoki from the University of Tokyo, with $Y$. Matsui from Okayama University, have brought it a good step nearer. Starting with the information that the polymorphic forms of pure silica consist exclusively of $\mathrm{SiO}_{2}$, they first calculate, from first principles, the force constants in clusters of the atoms and then, by molecular dynamics, the lattice constants of the four common polymorphs of silica ( $\alpha$-quartz, $\alpha$-cristobalite, coesite and stishovite - the two last first recognized in the debris from meteoritic impacts).

The authors thank two computer centres in Japan for access to computing machinery, although they do not say how many Cray-equivalent hours their calculations occupied (Phys. Rev. Lett. 61 871; 1988).

The calculation is not merely a taste of things to come but a pointer to the obstacles that remain, as well as being, in its own right, a considerable achievement. What the group has done is to invert the usual procedure in which force constants are calculated empirically from the properties of crystals. Their strategy is instead to calculate the force constants from first principles, for which purpose they must calculate the electronic structure of a complex of silicon and oxygen atoms.

The snag is that, even with the the price of Cray machines and their equivalents falling year by year, it remains beyond the capacity of computation to calculate the properties of a real crystal. So Tsuneyuki et al. start with the simplest of all relevant structures - the tetrahedral cluster $\mathrm{SiO}_{4}{ }^{4-}$.

But why $\mathrm{SiO}_{4}^{4-}$, not $\mathrm{SiO}_{4}$ ? Because $\mathrm{Si}$ readily surrenders electronic charge to oxygen atoms - the authors estimate 0.7 of an electron charge for every $\mathrm{SiO}$ bond, or close on two electron charges for those $\mathrm{O}$ atoms bound to two $\mathrm{Si}$ atoms. So, to ensure that their calculated structures are electrically neutral, the authors place electronic charges at points $1.65 \AA$ along the four tetrahedral axes of the cluster, neatly simulating in the process the electrostatic effects of the neighbouring oxygen ions found in structures such as quartz.

It would be wrong to say that, from this point, everything depends on what the programmers have done, but that is how the article reads. Tsuneyuki et al. essentially calculate the electronic structure of the cluster for various departures of the atoms from their mean positions. They use pre-calculated atomic orbitals in a Hartree-Fock self-consistent field procedure (one of the means of allowing for electron-electron interactions in manyelectron atoms).

In principle, there is every reason why the energy of the system should be quite a complicated function of the atomic positions. The surprise is that the parts of the energy surface so far calculated can be accurately represented by potential functions depending only on the distances between pairs of atoms in the cluster. Not surprisingly, one of these is a Coulomb term, another corresponds to the van der Waals attractive potential proportional to $r^{-6}$ where $r$ is the separation.

The molecular dynamics is made to seem just routine, no doubt because of the careful development of the appropriate programs by Japanese colleagues. Pressure and temperature are determined by the size of a repeating three-dimensional unit in a periodic lattice and by total kinetic energy respectively. The known structures of the four polymorphs of silica are each dealt with separately. In each case, the object of the exercise is to tell whether the polymorphs are dynamically stable, or that they are not spontaneously converted into each other, which all four prove to be. This is taken as an explanation of why they are all apparently stable under ordinary conditions even though, thermodynamically, they are metastable.

Given previous difficulties in calculating from first principles the properties of real solids, the results are extremely good. Unit cell dimensions turn out to be within 5 per cent of their measured values, bulk properties such as density and bulk modulus within 10 per cent. The agreement between calculated energy (enthalpy) of the polymorphs and the few measurements available is even better ( 1 per cent), but the authors say they could probably do even better if they allowed for the band structure of these polymorphs and the extent to which the bands are filled at different temperatures.

Several refinements remain to be explored. The chances are that three-body potentials and even more complicated variations of energy with configuration can be demonstrated by calculation and by comparison, for example, with measured specific heats. Evidently the treatment of charge transfer between $\mathrm{Si}$ and $\mathrm{O}$, while effective, is somewhat artificial and does not allow for cooperative configurations extending over more than one $\mathrm{SiO}_{4}^{4-}$ tetrahedron.

In themselves, of course, these results are not a fulfilment of the goal of calculating from first principles the crystal structure of a compound of which nothing is known except chemical composition. But that cannot be far away.

On the face of things, the most obvious artificially introduced variables are the known crystal structures of the polymorphs of silica, used as a starting point for the molecular dynamics. It should not be beyond the wit of Tsuneyuki and his colleagues, with the help of a few computer centres, to select from a listing of all possible structures of an arbitrary material those that can be considered plausible on crystallographic and other grounds.

Perhaps the most striking feature of this laconic paper is the contrast it presents with the state of the art of calculating the properties of solids as recently as a decade ago, when it seemed that even the use of force constants derived empirically from the measured properties of crystals did not regenerate, on calculation, the authentic properties of those same crystals. But the practical importance of the new calculation could be considerable, especially with the need to learn something of the crystal structure of materials that are either very small ('microdot' zero-dimensional quantum wells, for example) or which vary in composition over distances smaller than the limits of X-ray crystallography (such as doped semiconductors). In applying calculations to these needs, a demonstration of success can rank, psychologically, with the example set by those who first climbed Everest. 